\title{
Supply chain management et tableau de bord prospectif : à la recherche de synergies*
}

Joëlle MORANA,

Doctorante en Sciences de Gestion - CRET-LOG, Université de la Méditerranée (Aix-Marseille II)

\section{Gilles PACHÉ,}

Professeur en Sciences de Gestion à I'Université de Nice Sophia-Antipolis

Directeur de recherches au CRET-LOG, Université de la Méditerranée (Aix-Marseille II)

Face à un environnement de plus en plus complexe et turbulent, des publications relativement convergentes tendent à indiquer que l'efficacité d'une chaîne logistique globale se mesure à l'aune de son niveau de réactivité, de reconfiguration rapide des processus, d'élimination des gaspillages et d'intelligence.

Si un relatif consensus se dégage ainsi sur les facteurs-clés de succès d'un supply chain management, force est de reconnaître que peu de choses ont été dites et écrites sur les outils de pilotage permettant de les concrétiser.

L'article propose un certain nombre de pistes dans cette direction en s'appuyant sur un tableau de bord développé aux Etats-Unis, et dont le couplage avec le modèle World Class Logistics, issu de travaux menés par le Council of Logistics Management, semble prometteur aux auteurs, même si l'appropriation des indicateurs de résultats par les acteurs pose encore de nombreux problèmes.

Les années 1990 ont été marquées par des mutations majeures de l'environnement économique et institutionnel dans la plupart des pays occidentaux. Face à un consommateur de plus en plus volatil dans ses choix, les entreprises ont vu s'exacerber entre elles une vive concurrence par les prix et/ou par le service, au point que certains n'hésitent plus à parler d'hyper-compétition, un peu comme un enfant hyper-actif dont on a parfois du mal à contrôler des comportements agressifs !

Dans un tel contexte, l'obtention d'un avantage concurrentiel durable exige une remise en cause de schémas hérités du passé, au profit d'une flexibilité des structures organisationnelles, d'un accroissement de la réactivité et d'une aptitude à s'insérer dans un réseau de partenaires pour proposer le meilleur système d'offre possible.

Sans conteste, le dernier point nous paraît témoigner des nouvelles " règles" de la compétitivité en émergence. En effet, pour les entreprises industrielles et commerciales, l'heure est à la mobilisation efficace d'un ensemble de compétences dans l'univers stratégique qui les entoure. Beaucoup a déjà été
* Les auteurs remercient les lecteurs anonymes et le rédacteur en chef de Logistique \& Management pour leurs remarques et suggestions sur une première version de l'article. 
écrit sur ce thème [16], entre autres dans la perspective du resource-based management, mais sans toujours souligner l'importance que revêt dès lors la gestion des interfaces sur un double plan physique et informationnel. L'immense mérite des travaux menés depuis quelques années sur la démarche supply chain management (SCM) est d'avoir révélé l'importance majeure de l'implémentation de nouvelles architectures logistiques comme catalyseur des logiques de réseau.

Toutefois, tout ceci risque de rester assez flou (voire nébuleux !) pour les preneurs de décision en entreprise, notamment s'ils ne disposent pas d'un instrument de pilotage clair et rigoureux guidant l'action. L'objectif de l'article est de suggérer une piste de réflexion en ce sens, en proposant d'associer à la démarche SCM un outil développé en contrôle de gestion : le tableau de bord prospectif (TBP). Après avoir successivement présenté dans les deux premières parties les principes fondateurs du SCM et du TBP, nous verrons dans une troisième partie comment il est possible d'envisager leur "couplage", via le modèle World Class Logistics, ce couplage étant selon nous l'un des enjeux majeurs des années 2000. Une enquête de terrain menée auprès d'une multinationale du secteur des semi-conducteurs montrera toutefois que beaucoup reste à faire en matière de diffusion et d'utilisation d'indicateurs de résultats.

\section{Le SCM : avant tout une démarche de gestion}

Si l'on en croit de nombreux observateurs avertis, le seul "salut" d'une entreprise consiste aujourd'hui à améliorer sa réactivité, voire sa pro-activité, pour faire face de manière efficace aux mutations d'un environnement de plus en plus complexe et turbulent. La double traduction en est une maîtrise accrue des aléas, conduisant à une capacité de réponse en quasi-temps réel aux exigences des clients, ainsi qu'une introduction rapide de nouveaux produits, et ce avant les concurrents pour conserver un avantage pionnier. La logistique intégrée, plus connue sous son appellation anglo-saxonne de SCM, s'inspire de ce constat en cherchant à synchroniser un ensemble de flux physique, informationnel et financier qui, pilotés de manière intégrative, conduisent à améliorer la coordination et la compétitivité de tous les acteurs d'une chaîne afin de créer de la valeur pour le client final $[1,2,4]$.

\section{De nouvelles priorités émergentes}

C'est à Heskett [12,13], au milieu des années 1970, que l'on doit d'avoir le premier repéré la mutation d'une logistique opérationnelle et cloisonnée à une logistique intégrative. Constatant le passage d'une logique d'analyse en termes de coûts à une logique d'analyse en termes de profit, l'auteur met en évidence l'importance d'une parfaite coordination des flux de marchandises par les flux d'information dans la création d'utilités de lieu, d'endroit et de propriété. Ceci sous-tend un puissant processus d'interaction entre les intervenants de la chaîne d'opérations allant de l'aval (la demande à servir) vers l'amont (l'approvisionnement), à savoir l'entreprise manufacturière, mais aussi ses distributeurs, ses fournisseurs et ses prestataires de services logistiques... dont le rôle inducteur d'économies d'échelle (par mise en place de plates-formes communes de distribution) est rapidement identifié [13].

Posés il y a près de trente ans, les fondements modernes de la logistique d'entreprise ont vu depuis leur importance reconnue, entre autres au travers de quatre études successives conduites en Europe par le cabinet AT Kearney depuis 1982, et dont la dernière en date aboutit à la prise en compte pas à pas d'une démarche de type supply chain, phase ultime (?) de l'approche intégrative. Le constat principal des trois premières études est l'accent mis par les entreprises sur la réduction des coûts. Certes, si entre 1982 et 1987, elles ont d'abord connu une contraction des coûts de production et une augmentation des frais logistiques (qui sont passés de 11 à $21 \%$ de la valeur ajoutée), c'est surtout suite au développement des pratiques d'externalisation, aux dépens de l'intégration verticale, et à une croissance plus rapide des volumes à mettre en circulation que des volumes produits. En revanche, si l'on prend comme point de repère les ventes, les coûts logistiques ont chuté dans la plupart des entreprises européennes (-29\% entre 1982 et 1987), phénomène particulièrement remarquable au niveau du poste transport ( $-49 \%$ sur la même période).

Il ne faudrait pas en tirer la conclusion hâtive que la logique dominante, jusqu' au début des années 1990, est celle d'une stratégie de domination par les coûts, pour reprendre la fameuse terminologie portérienne, même si elle ne doit pas être négligée compte tenu d'un contexte économique largement récessionniste. Bien au contraire, la qualité de service proposée aux clients n'a cessé de croître 
depuis 1982. Remarquons toutefois qu'un assez faible nombre d'entreprises réussit à atteindre une "excellence logistique" en 1992, plus particulièrement en matière d'établissement de liens inter-organisationnels solides et de mise en place de programmes d'amélioration continue de la qualité de service.

La toute dernière étude, menée en 1997 dans 18 pays européens avec le soutien de l'ELA, semble indiquer une certaine inflexion. Les entreprises interrogées ont pris conscience d'une indispensable refonte des schémas logistiques. Mais l'étude précise aussi que si une majorité des entreprises concentre ses efforts sur la réduction des délais et l'amélioration de la qualité de service, de tels efforts restent insuffisants, entre autres parce que les mesures statiques et individuelles de la performance d'une supply chain ne permettent pas de réfléchir sur les modalités d'obtention d'un avantage concurrentiel durable. Problème d'ailleurs souligné récemment par Lebraty [19] qui s'interroge sur la pertinence de mesures "locales", au niveau d'une organisation, alors que des agencements optimaux pour elle-même ne le seront plus nécessairement au niveau de l'ensemble d'une chaîne, et inversement.

\section{Penser les architectures logistiques du futur}

Il n'en reste pas moins que l'étude AT Kearney-ELA de 1997 a pour intérêt de mettre en lumière les éléments critiques pour une meilleure profitabilité des entreprises impliquées dans une démarche SCM. Cette dernière insiste sur la gestion dynamique des interfaces, plutôt que sur un contrôle des activités fondées sur la propriété des actifs et l'intégration verticale, mais également sur son rôle de créateur de valeur (alors que la logistique avait tendance à privilégier une logique d'économie de coûts). Pour Mesnard et Dupont [21], à l'origine d'une contribution tout à fait stimulante sur le sujet, les piliers d'un SCM efficace sont de quatre ordres : la réactivité, l'agilité, l'efficience et l'intelligence.

Source d'avantage concurrentiel désormais fort connue, la capacité de réaction (ou de réponse) permet d'identifier puis satisfaire les demandes non prévues. En effet, dans une philosophie de time-based management, la faculté d'adaptation aux besoins du client devient primordiale, et justifie la mise en œuvre de nouveaux investissements matériels et immatériels. Dans ce cadre, comme le notent Gervais et Thenet [10], recourir à des technologies de l'information sophistiquées (télécommunications, procédures EDI, etc.) constitue une étape incontournable en vue de réduire les temps de transferts entre activités opératoires, ce qui accroît substantiellement la réactivité de la firme. Il en résulte une relation étroite avec l'aptitude à développer une certaine "agilité", en référence à une capacité technologique et managériale à reconfigurer rapidement un système d' offre en redéployant les ressources disponibles [21].

Cette dernière aptitude devient d'ailleurs déterminante pour Christopher [5] par rapport à une logique d'ajustement aux ruptures que peuvent rencontrer les organisations, puisqu'une chaîne logistique agile se définira en fonction de sa capacité d" "adaptation rapide, stratégique, et opérationnelle aux changements à grande échelle et imprévisibles de l'environnement". Toutefois, l'auteur considère que l'agilité d'une entreprise "implique la réactivité d'une extrémité de la chaîne à l'autre. Elle se focalise sur l'élimination des freins, qu'ils soient organisationnels ou techniques". En cela, il s'associe à Mesnard et Dupont [21], tout en soulignant qu' une chaîne logistique agile ne doit pas être confondue avec la notion de management au plus juste. Celle-ci fait appel à une approche JAT, souvent employée en liaison avec la fabrication, mais sans que l'entreprise soit toujours agile dans le fonctionnement de sa chaîne.

Si la réactivité et l'agilité jouent un rôle majeur dans l'émergence d'architectures logistiques performantes, deux autres aspects complémentaires ne sont cependant pas à minorer :

- Il s'agit en premier lieu d'être efficient dans sa capacité à éliminer systématiquement toute forme de gaspillage, et ce en cherchant à optimiser les frais des structures et des processus logistiques, les ressources engagées et les coûts externes pour l'environnement (éco-logistique intégrée). Cela signifie que, pour être durablement efficientes et efficaces, les entreprises devront notamment rechercher une maximisation des synergies entre les différentes supply chains dans lesquelles elles s'insèrent. L'application des techniques issues du reengineering répond, en partie, à ce critère d'efficience.

- En second lieu, c'est par son intelligence que l'organisation peut anticiper les attentes et besoins des clients, et se doter des ressources nécessaires pour espérer les satisfaire (vision globale de l'ensemble des flux de produits et d'informations associées). L'atteinte d'un tel objectif passe en 
particulier par l'existence d'échanges directs et le partage continu d'informations entre les parties prenantes concernées (clients, fournisseurs et prestataires de services). A ce titre, le benchmarking, "processus continu et systématique permettant de comparer (sa) propre activité en termes de productivité, de qualité et de pratiques avec les compagnies et les organisations qui représentent l'excellence" [15], apporte une aide précieuse qu'aucune entreprise ne peut négliger.

\section{Figure 1. SCM : la "pyramide magique"} (Mesnard et Dupont [21], p. 54)

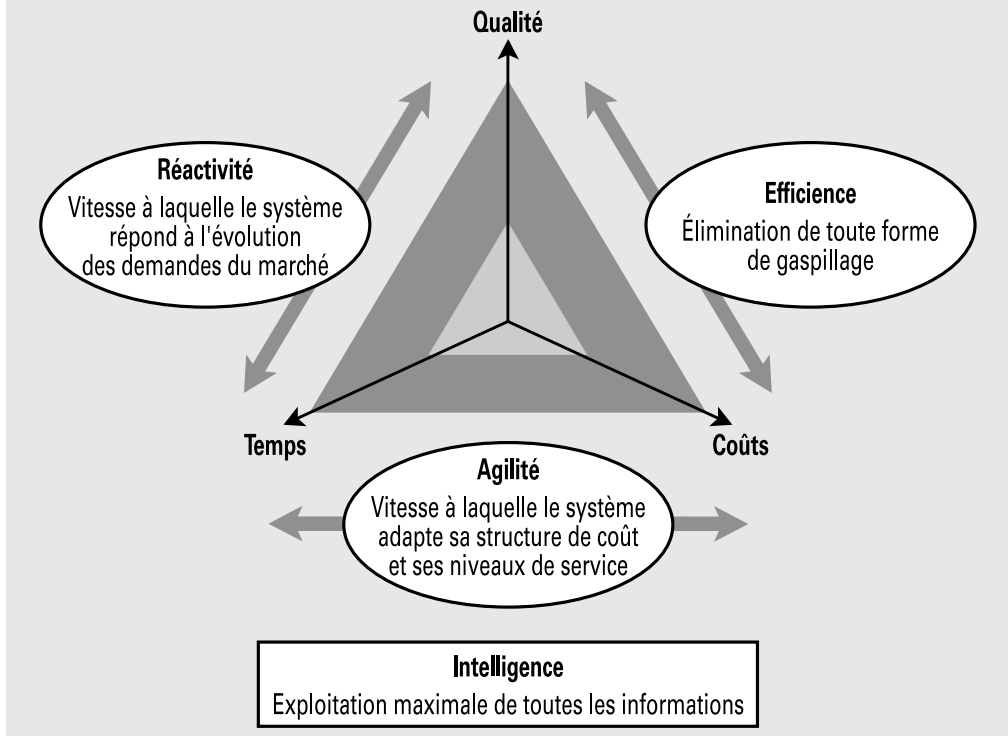

\section{Une "révolution" managériale}

Plus que tout, s'adapter à un environnement en profonde mutation, et valoriser les multiples "interférences stratégiques" qui lient entre elles les entreprises d'une même supply chain, exige de totalement repositionner la stratégie logistique. Composante de la politique générale, elle ne peut plus se contenter de penser la mise à disposition de moyens en vue de la conduite d'opérations de distribution physique, de production et d'approvisionnement. Ceci reviendrait effectivement à la réduire de nouveau à une sorte d'intendance au service de stratégies industrielles et/ou commerciales. Au contraire, la démarche SCM donne à la stratégie logistique de chacune des organisations impliquées un rôle majeur dans la génération d'activités supplémentaires et, par conséquent, dans la création de valeur que pourront se partager les parties prenantes. Un tel repositionnement demande à la fois de développer un nouvel état d'esprit dans l'entreprise et se doter des compétences nécessaires :
- Il n'est plus acceptable de considérer la logistique comme une fonction auxiliaire alors qu'elle se présente comme une véritable compétence-clé au sens de Hamel et Prahalad [11] : elle revêt une valeur significative aux yeux du client (même s'il en a peu conscience), favorise la différenciation par rapport aux concurrents et permet d'envisager, à partir d'elle, le développement futur de l'entreprise. A ce titre, la logistique, moins qu'un centre de coûts, devient une opportunité de création de valeur, et notamment de valeur économique offerte au client final.

- Faire prendre conscience des apports d'une démarche logistique est, certes, une avancée majeure. Encore faut-il en parallèle déployer dans chaque entreprise les processus, les systèmes d'information et les savoir-faire spécifiques sous-tendus par le SCM. Par delà l'implémentation d' outils de gestion plus ou moins sophistiqués, dont une certaine presse professionnelle tend régulièrement à se faire l'écho, ses principes sont fondés sur des compétences organisationnelles telles que l'écoute du client, la créativité, la discipline d'exécution et une remise en cause permanente des procédures [21].

Reconstruire la stratégie logistique en se fondant sur la mise en œuvre d'une supply chain est un processus complexe et difficile à mener, en particulier parce qu'il nécessite la remise en cause d'anciennes habitudes. Les relations nouées entre l'entreprise et ses fournisseurs de matières et composants, mais aussi ses prestataires de services logistiques, constituent une source d'avantage concurrentiel à condition de ne plus simplement raisonner sur la base d'un rapport de force où le prix est le facteur central de la négociation. Au contraire, l'objectif doit être de parvenir à des partenariats verticaux où les coûts, les investissements technologiques et les compétences humaines seront partagés. Plus largement, les partenaires doivent accepter de participer à l'élaboration d'un projet productif commun fondé sur des valeurs de réciprocité et de confiance, la démarche n'étant d'ailleurs pas exempte d'un calcul optimisateur [29].

Le décloisonnement et la transversalité dont est porteur un tel projet implique une évolution de la culture interne de chacune des parties prenantes pour provoquer le changement souhaité. Il implique également que l'on puisse disposer d'instruments de mesure et d'évaluation des performances. En effet, si la démarche logistique est inductrice de valeur 
pour un ensemble de partenaires, et donc de gains, comment procéder à sa répartition équitable entre eux ? Par delà un simple aspect financier, l'entreprise initiatrice d'une démarche SCM doit aussi être capable d'informer ses fournisseurs et prestataires de services logistiques sur des dimensions telles que la satisfaction des clients et l'engagement des employés, et ce afin de les mobiliser plus efficacement. Selon nous, le tableau de bord prospectif, en tant que modélisation des facteurs de performance s'appuyant sur des indicateurs financiers et non financiers, pourrait apporter une aide utile à la décision dans cette direction.

\section{Le TBP : avant tout un outil de gestion}

En provenance d'outre-Atlantique, le balanced scorecard ou encore tableau de bord prospectif (TBP) a été conçu à l'intention des diverses parties prenantes intéressées à la performance d'une entreprise, mais ne voulant pas prendre de décisions uniquement fondées sur des paramètres financiers. Cet outil de gestion, décrit par Kaplan et Norton [14], s'inspire entre autres d'une étude de l'American Institute of Certified Public Accountants qui souligne que "c'est le futur qui intéresse les analystes, mais les entreprises, aujourd'hui, ne fournissent d'informations que sur la performance passée. Ces informations apportent des indicateurs utiles sur ce que pourrait être la performance future, mais les analystes ont besoin de données prospectives" (in Kaplan et Norton [14]). En d'autres termes, il est nécessaire que l'entreprise de demain soit en mesure de fournir des indicateurs fiables sur la manière dont elle crée de la valeur future et sur sa capacité à relier des mesures de performance à une vision stratégique.

\section{Structuration des axes du TBP}

L'objet du TBP n'est pas de se substituer au système de mesure quotidien en vigueur dans l'entreprise, mais plutôt de formuler des indicateurs de manière à attirer l'attention des managers et des salariés sur les facteurs censés générer des avancées importantes dans la performance. Tout en gardant un œil, grâce aux indicateurs financiers, sur la performance à court terme, un tel tableau doit ainsi mettre en évidence les déterminants de l'amélioration de la performance concurrentielle à long terme par la définition d'indicateurs stratégiques. Cette démarche holistique, comme nous le verrons, peut sans doute être appliquée au niveau de la supply chain.

Kaplan et Norton [14] proposent de doter le TBP de quatre axes, à savoir les résultats financiers, les clients, les processus internes et l'apprentissage organisationnel, chacun des axes comptant entre quatre et sept indicateurs de performance. Certes, seulement quatre axes sont proposés à l'entreprise, mais à elle d'envisager le rajout d'autres si elle en ressent le besoin pour une meilleure gestion de ses données :

- L'axe "financier" sert de fil conducteur aux indicateurs des autres axes du TBP. L'objectif est que chaque indicateur sélectionné fasse partie d'une chaîne de relations de cause à effet dont le but ultime est d'améliorer la performance financière.

- L'axe "clients" identifie les segments de marché sur lesquels l'entreprise souhaite se positionner, ceux qui généreront le chiffre d'affaires nécessaire à la réalisation des objectifs financiers. Il s'agit d'un indicateur éclairant pour l'entreprise qui souhaite être le numéro un dans sa catégorie de produits et de services, et ce pour les groupes de clients qui l'intéressent.

- L'axe "processus internes" vise à améliorer l'ensemble des processus, depuis l'innovation jusqu'au service après-vente. Les objectifs et les indicateurs sont ici définis à partir de stratégies explicites visant à répondre aux attentes des actionnaires et des clients-cibles. Cette démarche séquentielle révèle généralement des processus nouveaux dans lesquels l'entreprise devra exceller à moyen terme.

- L'axe "apprentissage organisationnel" représente les moyens, ressources et facteurs qui permettent d' atteindre les objectifs établis sur les axes "financier", "clients" et "processus internes", sachant que ceux-ci identifient les domaines dans lesquels l'entreprise doit se distinguer pour améliorer sa performance.

\section{Un outil orienté sur l'action et la décision}

Le tableau 1 fournit un certain nombre d'indicateurs génériques pour chacun des quatre axes précédents. Plus généralement, l'apport majeur du TBP est de souligner qu'il est important de ne pas recourir uniquement à des investissements matériels et immatériels, qu'il s'agisse d'équipements ou de R\&D. De tels investissements sont certes indispensables, mais ne peuvent en aucun cas constituer une fin en soi. Pour améliorer leur compétiti- 
vité, les entreprises doivent aussi investir dans des infrastructures dont les trois principales composantes sont : le potentiel des salariés ; les capacités des systèmes d'information ; la motivation, la responsabilisation et l'alignement des objectifs entre ceux de l'entreprise et de ses acteurs internes.

Tableau 1. Des indicateurs pour chaque axe du TBP (Kaplan et Norton [14], p. 57)

\begin{tabular}{|l|l|}
\hline Axes & Indicateurs génériques \\
\hline Financier & Retour sur investissement et valeur ajoutée économique \\
\hline Clients & Satisfaction, fidélisation, part de marché et part de portefeuille clients \\
\hline Processus internes & Qualité, réactivité, coût de lancement de nouveaux produits \\
\hline Apprentissage & Satisfaction des salariés et système d'information \\
\hline
\end{tabular}

Durant les premières étapes du processus de mise en œuvre d'un TBP, il est important que le projet et la stratégie de la société soient clarifiés puis communiqués aux salariés, et enfin que des initiatives stratégiques "transversales" soient lancées [14]. On peut en escompter l'adoption de nouvelles valeurs et de nouvelles méthodes de travail axées sur la satisfaction du client, une qualité et une réactivité quotidienne mais aussi une innovation, un service et une responsabilisation accrus. En tant qu'outil orienté sur l'action et la décision, la création d'un TBP commence évidemment par un dialogue entre les différents acteurs internes de l'unité utilisatrice. Il sera construit comme un système vivant s'adaptant constamment à l'évolution de la structure qui choisit de l'intégrer, avec des mesures spécifiques à cette structure et non comme le reflet d'indicateurs choisis par des entreprises concurrentes.

Bref, le TBP est un outil de gestion stimulant qui souligne l'importance d'équilibres subtils entre court terme et long terme, critères financiers et non financiers, ou encore indicateurs majeurs et indicateurs marginaux. Du point de vue interne, il donne l'opportunité de signaler clairement quels sont les processus et les compétences pour lesquels l'entreprise doit être excellente. Mais l'outil peut-il être utilisé dans un autre contexte, celui d'un réseau de partenaires spécialisés qui vont collaborer durablement et étroitement entre eux en vue de concevoir, fabriquer, commercialiser et distribuer de nouveaux produits ? Il faut sans doute répondre par l'affirmative. En effet, dans ce cas, la performance concurrentielle de l'ensemble de la supply chain va dépendre des capacités d'interaction et de réactivité de ses membres, dont de simples indicateurs financiers ne peuvent rendre compte. De même, il sera nécessaire d'impliquer totalement les salariés dans un projet productif qui dépasse les frontières légales de leur propre entreprise d'appartenance, d'où la mise en œuvre de logiques d'apprentissage d'un tout nouveau type, sous peine de voir se multiplier des dysfonctionnements récurrents [24].

On retrouve au final, de manière implicite, de fortes convergences avec ce qui va permettre aux entreprises leaders sur un plan logistique de se différencier des autres entreprises, si l'on reprend les résultats de l'étude AT Kearney-ELA de 1997. En effet, les entreprises leaders y apparaissent plus réactives et en meilleure posture sur leur marché pour deux raisons essentielles :

- Elles utilisent plus largement des techniques avancées de contrôle de gestion, telles que l'ABC/ABM. Connaissant ainsi la réalité de leurs coûts logistiques, elles sont capables de mieux cibler leurs efforts et leurs plans de progrès, par produit ou par client.

- Elles acceptent la remise en cause permanente de leur organisation, que cela soit par une remise à plat périodique (principe du reengineering) et/ou par une fine observation des pratiques et des performances de la concurrence (principe du benchmarking). Ce faisant, les entreprises leaders s'inscrivent dans des logiques de flexibilité et d'adaptation au bénéfice du client final.

\section{Premiers pas (difficiles) vers un processus de "couplage"}

Le couplage entre SCM et TBP constitue, selon nous, une piste d'investigation de première importance dès lors que l'objectif est, comme le précise Vera [28], de ne négliger aucun des facteurs susceptibles d'avoir une influence sur le résultat d'une organisation. Ceci apparâit d'autant plus urgent qu'une multiplicité d'acteurs est en jeu. Le modèle World Class Logistics (WCL), issu des travaux du Council of Logistics Management aux Etats-Unis et dont une excellente application est fournie dans Estampe et al. [8], servira de point d'appui à la démarche de couplage entreprise, sa dimension étant - pour l'instant - de nature exploratoire (voir figure 2). A la suite de Brosset [3], nous pensons que "de nombreux programmes de supply chain commencent par reconcevoir les processus, à partir de quoi on élabore un tableau de bord plus ou moins complet destiné à mesurer les progrès (...). Pourquoi ne pas commencer par définir le tableau de bord puis l'utiliser pour provoquer et mesurer le progrès ?". 
Figure 2. SCM et TBP : lieu d'ancrage entre objectifs convergents

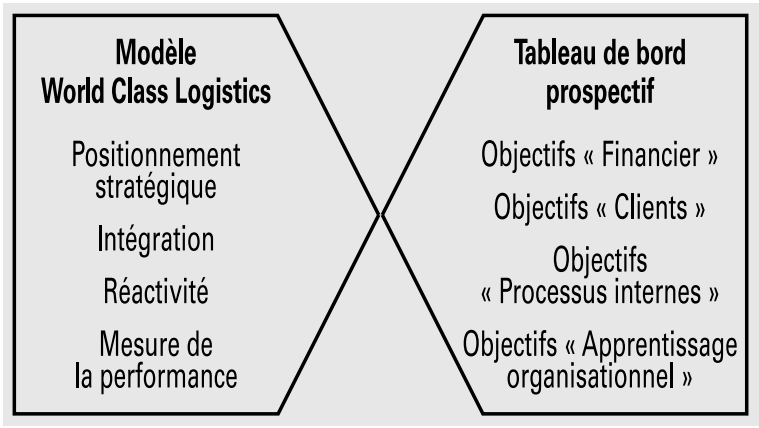

\section{Les points de rencontre}

De façon synthétique, le modèle WCL regroupe les facteurs-clés de succès d'une politique de chaîne logistique au travers de quatre domaines de compétences : le positionnement stratégique, l'intégration, la réactivité et la mesure de la performance. Ces quatre domaines sont à mettre en parallèle avec les quatre axes d'évaluation du TBP évoqués au paragraphe précédent. Le rapprochement du modèle WCL (annexe 1) et du TBP (annexe 2) fournit un certain nombre d'indicateurs multidimensionnels de performance pour une meilleure coordination inter-organisationnelle, entendue comme l'ensemble des liaisons verticales existant entre les différents intervenants de la chaîne globale qui va du fournisseur au client final, en passant par les industries de transformation, le canal de distribution et les prestataires de services logistiques [25].

- Le positionnement stratégique. Il est défini par les choix de différentes orientations stratégiques et structurelles en vue d'optimiser les opérations et activités logistiques. Son application touche à la fois à l'axe "financier" (mise en œuvre d'objectifs financiers et commerciaux), à l'axe "clients" (ajustement mutuel entre les partenaires pour améliorer la qualité de service rendue) et à l'axe “apprentissage organisationnel" (développement adapté des ressources humaines) du TBP.

- L'intégration. Il s'agit des moyens mis en place pour synchroniser l'ensemble de la chaîne logistique. Le critère de partage de l'information nous semble le plus important puisqu'une volonté d'échange de données à caractère technique, financier, opérationnel et stratégique représente "l'élément différenciateur de l'entreprise de l'ère informationnelle actuelle" [14]. Les autres critères se connectent aux quatre axes du TBP. Ainsi, l'amélioration des pré- visions de performance peut se concevoir au travers du reengineering des procédures. Les investissements en systèmes d'information, la compatibilité des informations transmises et la mise en place de procédures communes se conjuguent avec les indicateurs "processus internes : exploitation et innovation". Quant à l'unification de la supply chain et l'adhésion des hommes, ils trouvent une filiation directe avec l'axe " clients" et l'axe "apprentissage organisationnel".

- La réactivité. Elle est perçue comme la faculté à conserver une adéquation entre la performance de l'entreprise et l'adaptation aux besoins du client. Les trois critères (veille, adaptabilité et flexibilité) se combinent parfaitement avec trois indicateurs "processus internes" du TBP (responsabilisation des chargés de clientèle, fidélisation des clients et acquisition d'un savoir-faire sur les technologies du futur).

- La mesure de la performance. Elle permet d'évaluer le plus finement possible la performance globale de la chaîne logistique multi-acteurs. Les choix d'indicateurs, l'évaluation du processus supply chain et le benchmarking se retrouvent de manière identique dans la composition même du TBP. Pour les deux autres, l'analogie s'établit au niveau de l'objectif "processus internes : exploitation".

\section{Une réalité parfois loin des espérances}

Afin d'évaluer les perspectives de mise en œuvre d'indicateurs de performance dans un contexte SCM, il nous a paru instructif de procéder à une analyse préalable de l'existant en référence à un cas réel d'entreprise. Il s'agit en l'occurrence d'une multinationale du secteur des semi-conducteurs, impliquée depuis 1996 dans une démarche SCM pour tout ce qui concerne l'assemblage des puces électroniques dans des boîtiers. Avec le soutien actif de la Direction Générale, une enquête par questionnaire a été menée durant l'été 1999 auprès de 195 managers des six filiales françaises relevant des services marketing, après-vente, approvisionnement, fabrication, achats, comptabilité, contrôle de gestion, informatique et qualité. Deux types d'échelles de mesure ont été utilisés : une échelle de type dichotomique (oui, non, ne sait pas) permettant d'évaluer le degré de connaissance par les acteurs des méthodes, outils et processus en relation avec le SCM ; une échelle de type Likert à six points permettant d'évaluer leur position sur des thèmes relatifs à 
l'amélioration des performances, à l'implication, à la mobilisation des compétences, etc.

L'investigation s'est effectuée selon le principe d'une "coupe instantanée" dans la mesure où l'enquête, se déroulant volontairement sur une période d'un mois, a permis d'obtenir une vision spontanée des acquis de la démarche SCM par les différentes personnes interrogées. Sur les 195 questionnaires envoyés, nous en avons recueilli 54 au final, soit un taux de réponse tout à fait satisfaisant de $27.7 \%$. La présence de 31 questionnaires partiellement remplis tend toutefois à indiquer que de nombreux acteurs internes ont beaucoup de mal à appréhender la complexité d'une action stratégique de nature logistique touchant aux processus, ce qui correspond ici aux analyses de Fabbe-Costes [9] sur le sujet. De fait, seule une analyse statistique univariée a été conduite. Malgré son caractère exploratoire et quelque peu réducteur, elle apporte un certain nombre d'informations intéressantes dans la perspective d'un couplage optimal entre SCM et TBP. Le lecteur intéressé trouvera l'ensemble des données recueillies puis traitées dans le travail de Morana [22] dont nous présentons ici quelques éléments.

Le tableau 2 regroupe un certain nombre d'indicateurs de résultats pour lesquels une entreprise doit exceller dans une logique SCM, et auxquels ont été confrontées les personnes interrogées ("avez-vous connaissance d'indicateurs de résultats portant sur... ?"). Ils découlent d'un effort d'adaptation de différents indicateurs de performance pour refléter la perspective logistique en permettant de déterminer si le répondant en a une relative connaissance, qu'il s'agisse d'une diminution des coûts, d'une réduction des délais de livraison ou, enfin, d'une amélioration de la qualité de service rendu au client. En cela, nous empruntons directement aux objectifs "finan-

Tableau 3. Impacts de la démarche SCM selon les personnes interrogées

\begin{tabular}{|c|c|}
\hline Items & Moyenne \\
\hline $\begin{array}{l}\text { Êtes-vous tout à fait d'accord (6), plutôt d'accord (5) légèrement d'accord } \\
\text { (4), légèrement en désaccord (3), plutôt en désaccord (2), tout à fait en dé- } \\
\text { saccord (1) avec les affirmations suivantes : } \\
\text { - La mise en place d'une démarche SCM a amélioré la qualité des rap- } \\
\text { ports entre services } \\
\text { - La mise en place d'une démarche SCM a amélioré la qualité des rap- } \\
\text { ports entre sites } \\
\text { - La mise en place d'une démarche SCM a amélioré la qualité des rap- } \\
\text { ports avec les fournisseurs } \\
\text { - La mise en place d'une démarche SCM a amélioré la qualité des rap- } \\
\text { ports avec les prestataires logistiques } \\
\text { - La mise en place d'une démarche SCM a amélioré la qualité des rap- } \\
\text { ports avec les clients }\end{array}$ & $\begin{array}{l}4.7 \\
3.5 \\
3.5 \\
4.3 \\
2.4\end{array}$ \\
\hline
\end{tabular}

cier" (coûts), "clients" (service) et "processus internes" (commande et facturation) du TBP présenté en annexe 2, ainsi qu'aux indicateurs de mesure de la performance du modèle WCL présenté en annexe 1.

Force est d'admettre que les résultats obtenus sont extrêmement décevants : moins de $10 \%$ des personnes interrogées ont répondu par la positive à sept des neuf questions. Seuls les indicateurs de résultats concernant la réduction des coûts relatifs à la gestion des stocks $(25.8 \%)$ et la réduction des délais de livraison entre le fournisseur et l'entreprise (19.4\%) obtiennent un score un peu plus convenable. En tout état de cause, ceci reste insuffisant pour affirmer que les acteurs internes d'une entreprise, pourtant impliquée dans une démarche SCM depuis plus de trois ans, ont accès aux indicateurs permettant de mesurer l'évolution des performances et, indirectement, la réactivité de l'organisation.

\section{Tableau 2. Indicateurs de résultats per- mettant d'évaluer les impacts d'une dé- marche SCM}

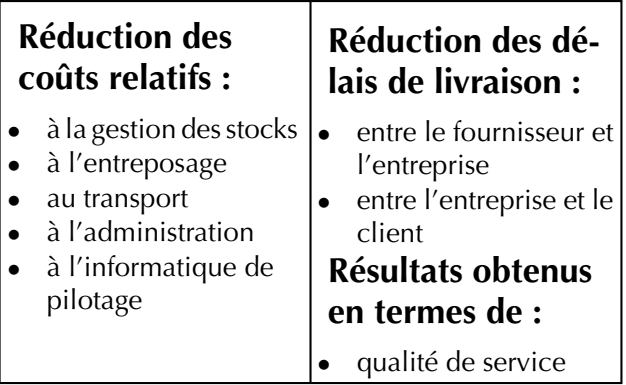

Ce point est d'ailleurs conforté par le fait que seulement une personne interrogée sur cinq connaît l'existence d'un supply chain manager, à quelque niveau que ce soit (local, national, européen). Et moins de $10 \%$ des répondants disent avoir été formés au processus de mise en place d'un SCM ! Il n'est donc pas surprenant que sur une échelle allant de 1 (pas du tout d'accord) à 6 (tout à fait d'accord), ceux-ci se posent des questions sur l'impact réel de la démarche SCM en matière d'amélioration de la qualité des rapports avec les prestataires logistiques (4.3) et avec les fournisseurs (3.5), et plus encore en matière d'amélioration de la qualité des rapports avec les clients (2.4), même si la coordination entre services semble y avoir gagné (4.7). Selon nous, de tels résultats indiquent, à la suite de Lawrence et Lorsch [18], que l'affirmation progressive de construits relationnels de type SCM exige en préalable une responsabilisation formelle (et clairement perçue) au niveau des tâches de coordination. 
Notre enquête exploratoire démontre, en tout état de cause, que la prise de conscience de l'importance d'un SCM demeure encore problématique, notamment faute d'une implication des individus autour d'un tel projet productif. L'absence d'adhésion des hommes, inquiétante en matière d'intégration, trouve sans doute ses racines dans la défaillance du système d'information, et plus particulièrement dans son incapacité à fournir des indicateurs (et un tableau de bord) rendant compte des résultats des efforts consentis par les uns et les autres, par exemple en vue d'accroître la satisfaction des clients. Certes, l'étude de cas ici menée ne revendique pas une absolue représentativité, entre autres parce que le nombre de répondants est finalement assez réduit. En revanche, il est symptomatique de constater que bien après ses premiers pas dans l'entreprise étudiée, la démarche SCM a du mal à être reconnue et comprise comme un enjeu stratégique majeur. Rien d'étonnant à cela, si l'on en croit Tixier et al. [26], car toute politique logistique, pour être efficiente, doit d'abord être explicite aux yeux de tous.

\section{Et demain?}

En résumé, la conduite d'un SCM nécessite le développement d'outils permettant d'identifier rapidement les éléments-clés qui offriront la possibilité à l'organisation d'être réactive, agile, efficiente et intelligente. L'élaboration d'un système de mesure multidimensionnel applicable à cette philosophie intégrative de la gestion globale des flux au sein d'un circuit, pour reprendre Cooper et al. [6], doit aboutir à l'établissement d'un point de rencontre entre les multiples attentes de chaque acteur des différentes filières économiques. Même si notre article se veut une première réflexion dans cette direction, en n'ignorant pas les difficultés d'implémentation, il souligne que des convergences fortes existent entre le modèle WCL et le TBP. A ce titre, leur couplage apparaît d'ores et déjà stimulant d'un double point de vue :

- au plan conceptuel, avec la (re)connaissance d'une nouvelle démarche de management, dont les implications vont bien au-delà d'une logistique traditionnelle focalisée sur le pilotage des flux plutôt que sur l'intégration des activités [2, 17],

- au plan managérial, avec l'élaboration d'un système de mesure devant permettre aux partenaires de la supply chain d'évaluer pourquoi ils auront objectivement avantage à opter pour des relations du type "gagnant-gagnant" plutôt que du type "gagnant-perdant" [4, 23].
Pour parvenir à l'élaboration d'un tableau de bord supply chain, beaucoup d'efforts restent néanmoins à consentir. Peu d'entreprises sont, semble-t-il, culturellement prêtes à sacrifier leur propre efficience au profit d'une optimisation globale, sachant que d'autres partenaires pourraient peut-être tirer un plus grand bénéfice à court terme d'une participation au réseau d'échanges. En outre, il n'est pas possible de faire l'impasse sur le contexte concurrentiel dans lequel va se mouvoir la supply chain, chaque cas de figure exigeant un instrument de mesure adapté [27]. Ainsi, dans un environnement de price competition, le TBP devra privilégier des critères liés à la maîtrise des coûts par chacun des partenaires, tandis qu'en présence d'une stratégie d'extension du marché par accroissement du service aux clients, c'est plutôt le critère de maîtrise des temps de réponse qui sera le plus adapté. Bref, loin de promouvoir une "pensée unique", le rapprochement entre modèle WCL et TBP doit tenir compte de facteurs de contingence et déboucher sur un outil d'aide à la décision à la fois souple et modulaire, dont les divers acteurs pourront aisément s'approprier grâce à une formation et une information suffisantes.

\section{Conclusion}

En l'état actuel, il serait maladroit de minimiser, ou pis d'ignorer, les écueils à l'établissement d'un SCM. Le principal blocage est sans doute dû à la volonté encore affirmée par certains managers de s'opposer à des décloisonnements fonctionnels et inter-organisationnels trop rapides. Il est vrai que l'entreprise au sens large reste encore un lieu de pouvoir, ce dernier se mesurant souvent par le contrôle sans partage d'un “ territoire" bien identifié. En leur temps, Crozier et Friedberg [7] ont explicitement mis en exergue cette approche politique des organisations entendues comme construits sociaux, où la prise de décision résulte de multiples négociations et de subtils compromis entre groupes d'individus. Or, la situation a-t-elle radicalement changé aujourd'hui ?

Mais l'absence d'indicateurs adaptés de mesure des performances constitue un frein tout aussi puissant [20]. En effet, si les partenaires d'une même supply chain sont dans l'incapacité de connaître les gains issus de la démarche, et surtout leur clé de répartition, le risque paraît grand de les voir se désimpliquer, voire se retirer à moyen terme du projet productif. Selon nous, un couplage du SCM et du 
TBP, débouchant sur un outil de mesure et de pilotage plus " consensuel ", devrait favoriser une clarification de la situation (et de la contribution) de chacun des acteurs, et ainsi offrir la possibilité de penser une véritable gestion transversale. Ne pas admettre que le cheminement pour $y$ arriver sera long et sinueux risque cependant de conduire à de cruelles désillusions...

\section{Références bibliographiques}

1. Aurifeille, J.-M., Colin, J., Fabbe-Costes, N., Jaffeux, C., et Paché, G. (1997), Management logistique : une approche transversale, Litec, Paris.

2. Bechtel, C., et Jayaram, J. (1997), Supply chain management : a strategic perspective, International Journal of Logistics Management, Vol. 8, $\mathrm{n}^{\circ} 1$, pp. 15-35.

3. Brosset, P. (1999), Les vices cachés des supply chains, L'Expansion Management Review, $\mathrm{n}^{\circ} 94$, pp. 90-93.

4. Christopher, M. (1998), Logistics and supply chain management, Pitman Publishing, London, 2nd ed.

5. Christopher, M. (1999), Les enjeux d'une supply chain globale, Logistique \& Management, Vol. 7 , $\mathrm{n}^{\circ}$ 1, pp. 3-6.

6. Cooper, M., Lambert, D., et Pagh, J. (1997), Supply chain management : more than a new name for logistics, International Journal of Logistics Management, Vol. 8, $\mathrm{n}^{\circ}$ 2, pp. 1-18.

7. Crozier, M., et Friedberg, E. (1977), L'acteur et le système : les contraintes de l'action collective, Editions du Seuil, Paris.

8. Estampe, D., Harreguy, A., Maldes, V., Mamère, M., Nougaret, S., et Truin, B. (1999), La performance supply chain des acteurs du secteur automobile en France, Logistique \& Management, Vol. 7, $\mathrm{n}^{\circ} 1$, pp. $15-22$.

9. Fabbe-Costes, N. (1997), L'intervention de la logistique dans la formulation/mise en acte de la stratégie en milieu complexe, in Avenier, M.-J. (éd.), La stratégie "chemin faisant", Economica, Paris, pp. 239-267.

10. Gervais, M., et Thenet, G. (1998), Planification, gestion budgétaire et turbulence, Finance Contrôle Stratégie, Vol. 1, n 3 , pp. 57-84.

11. Hamel, G., et Prahalad, C.K. (1994), Competing for the future, Harvard Business School Press, Boston (MA).

12. Heskett, J. (1973), Sweeping changes in distribution, Harvard Business Review, Vol. 51, $\mathrm{n}^{\circ} 2$, pp. 123-132.

13. Heskett, J. (1977), Logistics-Essential to strategy, Harvard Business Review, Vol. 55, nº 6, pp. 85-96.
14. Kaplan, R., et Norton, D. (1998), Le tableau de bord prospectif. Pilotage stratégique : les 4 axes du succès, Les Editions d'Organisation, Paris.

15. Karlöf, B., et Ostblom, S. (1995), Le benchmarking : un indicateur d'excellence en matière de qualité et de productivité, Travail et Méthodes, $\mathrm{n}^{\circ} 519$, pp. 3-17.

16. Koenig, G. (1996), Management stratégique : paradoxes, interactions et apprentissages, Nathan, Paris.

17. Lambert, D., Cooper, M., et Pagh, J. (1998), Supply chain management : implementation issues and research opportunities, International Journal of Logistics Management, Vol. 9, $\mathrm{n}^{\circ}$ 1, pp. 1-13.

18. Lawrence, P., et Lorsch, J. (1989), Adapter les structures de l'entreprise : intégration ou différenciation, Les Editions d'Organisation, Paris.

19. Lebraty, J. (2000), S'intéresser à la logistique : un pari scientifique, managérial et pédagogique, in Fabbe-Costes, N., Colin, J., et Paché, G. (éds.) Faire de la recherche en logistique et distribution?, Vuibert-Fnege, Paris, pp. 5-28.

20. Martin, A., et Landvater, D. (1997), Principes et perspectives du réapprovisionnement continu au sein de la supply chain, Editions Aslog, Paris.

21. Mesnard, X., et Dupont, A. (1999), Votre logistique est-elle à la pointe ?, L'Expansion Management Review, $\mathrm{n}^{\circ} 94$, pp. 52-58.

22. Morana, J. (1999), Le supply chain management face aux acteurs internes d'une organisation : étude exploratoire. Le cas d'une multinationale du secteur de la haute technologie, Mémoire de DEA en Sciences de Gestion, Institut d'Administration des Entreprises, Aix-en-Provence, octobre.

23. Munson, C., Rosenblatt, M., et Rosenblatt, Z (1999), The use and abuse of power in supply chains, Business Horizons, Vol. 42, n 1, pp. 55-65.

24. Paché, G. (1996), L’entreprise en réseau entre mythes et réalités, Gestion 2000-Management \& Prospective, Vol. 12, $\mathrm{n}^{\circ} 1$, pp. 33-50.

25. Paché, G., et Sauvage, T. (1999), La logistique : enjeux stratégiques, Vuibert Entreprise, Paris, 2 éd.

26. Tixier, D., Mathe, H. et Colin, J. (1996), La logistique d'entreprise : vers un management plus compétitif, Dunod, Paris.

27. Van Hoek, R. (1998), "Measuring the unmeasurable" - measuring and improving performance in the supply chain, Supply Chain Management, Vol. $3, \mathrm{n}^{\circ} 4$, pp. 187-192.

28. Vera, J. (1998), Vers un système intégré de mesure de la performance globale, Working Paper \# 512, Institut d'Administration des Entreprises, Aix-enProvence, février.

29. Williamson, O. (1993), Calculativeness, trust and economic organization, Journal of Law and Economics, Vol. 36, $\mathrm{n}^{\circ}$ 2, pp. 453-486. 


\section{Annexe 1}

\section{Les quatre indicateurs du modèle World Class Logistics (d'après Estampe et al. [8], p. 16).}

\section{A) Positionnement stratégique}

Le positionnement stratégique est défini par les choix d'orientations stratégiques et structurelles pour optimiser les opérations logistiques. Ce domaine regroupe les critères suivants :

- La stratégie logistique est définie par la mise en place d'objectifs financiers, commerciaux et de choix d'implantation ainsi que les moyens de les atteindre.

- La supply chain est la synchronisation des ressources de plusieurs partenaires le long de la chaîne logistique.

- Les infrastructures/réseau concernent la structuration et la répartition des ressources physiques.

- L'organisation des hommes porte sur la structuration et l'implication des ressources humaines.

\section{B) Intégration}

L'intégration est définie par les moyens mis en place pour synchroniser l'ensemble de la chaîne logistique. Elle regroupe les critères suivants :

- L'unification de la supply chain montre la capacité à développer des relations de coopération avec les autres entreprises à travers la chaîne logistique.

- Les systèmes d'information regroupent les investissements en matériels, logiciels et réseaux ainsi que leur adaptation pour faciliter les processus et les échanges d'information sur la chaîne.

- Le partage de l'information illustre la volonté d'échanger des données essentielles à caractère technique, financier, opérationnel et stratégique.

- La compatibilité met en évidence la capacité de l'entreprise à échanger des informations dans un format approprié, réactif et facilement utilisable sur la chaîne logistique.

- La standardisation implique la mise en place de politiques et de procédures communes pour faciliter et améliorer les opérations logistiques.

- La simplification consiste à faire du reengineering des procédures afin d'en améliorer l'efficacité.

- L'adhésion des hommes montre leur acceptation des politiques et des procédures opérationnelles.

\section{C) Réactivité}

La réactivité est la faculté à conserver une adéquation entre la performance de l'entreprise et l'adaptation aux besoins du client. Trois critères font partie de ce domaine :

- La veille est la capacité à rester attentif aux besoins changeants des clients.

- L'adaptabilité permet de diminuer les temps de réponse aux demandes exceptionnelles des clients.

- La flexibilité met en évidence la capacité à s'adapter aux circonstances inattendues.

\section{D) Mesure de la performance}

La mesure de la performance permet d'évaluer la performance de la chaîne logistique. Elle regroupe les critères suivants :

- Les choix d'indicateurs internes portent sur la gestion des actifs, les coûts, le service client, la productivité et la qualité.

- L'évaluation du processus supply chain permet d'affiner la mise en place des indicateurs le long de la chaîne logistique.

- Le benchmarking permet la comparaison des mesures et des processus avec les capacités des meilleurs. 


\section{Annexe 2.}

Exemple d'un tableau de bord prospectif à partir de cas réels (d'après Kaplan et Norton [14], p. 164 et pp. 246-247)

\begin{tabular}{|c|c|c|}
\hline \multirow{2}{*}{ Objectifs stratégiques } & \multicolumn{2}{|c|}{ Indicateurs stratégiques } \\
\hline & Indicateurs a posteriori & Indicateurs avancés \\
\hline \multicolumn{3}{|l|}{ Objectifs " financiers " } \\
\hline $\begin{array}{l}\text { - Atteindre une valeur ajoutée } \\
\text { économique positive }\end{array}$ & & \\
\hline - Améliorer la rentabilité & Retour sur investissement & \\
\hline $\begin{array}{l}\text { - Améliorer les prévisions de } \\
\text { performance }\end{array}$ & & Mix des sources de revenus \\
\hline - Atteindre une croissance de X\% pour & Augmentation du $C A$ & \\
\hline _ Réduire les coûts & Evolution des coûts & \\
\hline \multicolumn{3}{|l|}{ Objectifs "clients" } \\
\hline $\begin{array}{l}\text { - Créer des partenariats à forte valeur } \\
\text { ajoutée avec X\% des clients }\end{array}$ & Part de marché par segment & Elargir la relation de clientèle \\
\hline $\begin{array}{l}\text { - Améliorer la qualité et le service } \\
\text { pour approcher un taux de } \\
\text { satisfaction de } 100 \%\end{array}$ & Conservation des clients & Enquête de satisfaction \\
\hline \multicolumn{3}{|l|}{ Objectifs " processus internes" } \\
\hline \multicolumn{3}{|l|}{ Cycle d'exploitation: } \\
\hline & Taux d'erreurs dans la division ou le & \\
\hline $\begin{array}{l}\text { - Tendre vers le zéro défaut dans les } \\
\text { opérations }\end{array}$ & $\begin{array}{l}\text { département } \\
\text { Taux de défauts en pièces par million }\end{array}$ & \\
\hline $\begin{array}{l}\text { - Jouer sur le service pour restaurer } \\
\text { l'image de marque }\end{array}$ & & Temps consacré aux clients \\
\hline \multicolumn{3}{|l|}{ Cycle d'innovation : } \\
\hline $\begin{array}{l}\text { - Mieux connaître les marchés de } \\
\text { l'entreprise }\end{array}$ & CA généré par les nouveaux produits & Cycle de développement des produits \\
\hline \multirow{3}{*}{\multicolumn{3}{|c|}{$\begin{array}{l}\text { - Acquérir un savoir-faire sur les } \\
\text { technologies du futur } \\
\text { - Développer des solutions à valeur } \\
\text { ajoutée (nouveaux marchés) } \\
\text { - Améliorer l'image de marque auprès } \\
\text { des clients (fidélisation) }\end{array}$}} \\
\hline & & \\
\hline & & \\
\hline \multicolumn{3}{|l|}{ Objectifs "apprentissage organisationnel" } \\
\hline \multicolumn{3}{|l|}{$\begin{array}{l}\text { - Réorienter les compétences du } \\
\text { personnel }\end{array}$} \\
\hline $\begin{array}{l}\text { - Créer les conditions d'un climat } \\
\text { dynamique }\end{array}$ & Satisfaction des salariés & Alignement des objectifs personnels \\
\hline - Relier primes et performance & CA par salarié & \\
\hline $\begin{array}{l}\text { - Développer les systèmes } \\
\text { d'information }\end{array}$ & & $\begin{array}{l}\text { Ratio de disponibilité des informations } \\
\text { stratégiques }\end{array}$ \\
\hline
\end{tabular}

